Ann. Sci. forest., 1980, 37 (4), 291-297.

\title{
Etudes entreprises sur Cryptococcus fagi Baersp. en Haute-Normandie
}

\author{
C. B. MALPHETTES \\ avec la collaboration technique de Claudine Courtin et Sylvie Augustin \\ Station de Zoologie ef de Biocoenotique Forestières, I.N.R.A., \\ Centre de Recherches d'Orléans, \\ Ardon, 45160 Olivet, France
}

\section{Résumé}

L'auteur soumet à la discussion ses premiers résultats des études concernant la dynamique de la Cochenille du Hêtre.

La fertilité est d'autant plus élevée que la densité de la population de la cochenille est plus forte sur le tronc.

Le nombre des cochenilles fixées est bien plus faible que le nombre des cochenilles présenies sur la même surface d'écorce.

Un dispositif expérimental a été installé en 1978 pour suivre l'évolution de cet insecte en HauteNormandie au cours des prochaines années. Il apparaît que les populations, dans un même massif, sont bien plus faibles quand le sol est calcaire.

\section{Introduction}

«Le texte qui vous est présenté maintenant, est destiné à faire connaître quelques résultats de nos éfudes en cours, pour permettre une discussion au cours du colloque. Ce n'est en aucun cas, l'expression définitive de nos conclusions sur ce sujet. C'est pourquoi il n'y a pas de bibliographie. »

La maladie de l'écorce du hêtre a commencé à faire des ravages depuis une dizaine d'années, dans les hêtraies de Haute-Normandie.

Depuis le début des attaques, il y a eu une période d'accalmie ; mais il n'y a pas eu de régression à proprement parler. Comme les hêtraies de cette région sont des peuplements pratiquement purs, comme vous pourrez le constater, les dégâts sont considérables ; les données suivantes le montrent à l'évidence : ce sont des mètres cubes de bois atteints par cette calamité dans la Forêt Domaniale de Lyons. 


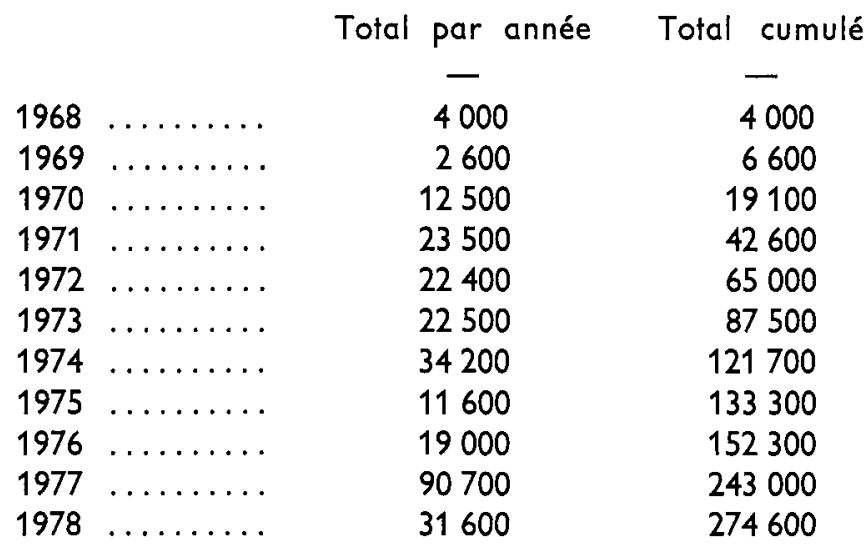

Ceci représente, en moyenne, plus de la «possibilité » de la forêt, qui se situe vers 20 à $24000 \mathrm{~m}^{3} / \mathrm{an}$. Du fait que les autres mesures de sylviculture ne doivent pas être abandonnées, cela conduit à un certain appauvrissement des peuplements. Certaines parcelles, qui n'ont pas encore atteint l'âge de la mise en régénération, n'ont déjà plus un nombre suffisant d'arbres pour assurer un ensemencement naturel. A cela s'ajoute le travail qui est nécessaire pour, dans les peuplements, retrouver les arbres atteints irréversiblement, et qu'il faut abattre rapidement, afin d'éviter une trop grande dépréciation.

Le problème posé par la cochenille Cryptococcus fagi Baersprung est devenu notre axe principal de travail depuis 1978. Nous vous présentons quelques indications concernant la dynamique de la cochenille et l'évolution de cette maladie dans plusieurs forêts de Haute-Normandie.

\section{I. - Dynamique de la population de Cryptococcus fagi}

En forêt de Lyons en 1978, deux études onf été menées successivement. Nous avons tout d'abord observé la fécondité réelle, puis l'implantation des jeunes larves à l'enirée de l'hiver.

\section{1. - Fécondité}

\subsection{Méthodologie.}

Des échantillons ont été prélevés en deux endroits de la forêt, l'un au nord, l'autre au sud, dans des peuplements âgés de 90 à 120 ans ou de 60 à 90 ans. Dans tous les cas, il s'agit de peuplements purs de hêtres. A chaque fois, douze arbres ont été étudiés pour une surface de 100 à 120 ha. En outre, les arbres choisis portaient soit de fortes populations de cochenilles, soit de faibles populations.

Pour suivre la fécondité sur chaque arbre, une rondelle d'écorce d'un diamètre de $10 \mathrm{~mm}$ a été prélevée avec l'aide d'un emporte-pièce.

Après stockage au congélateur, les échantillons sont examinés sous la loupe binoculaire pour compter femelles ef œufs. 


\subsection{Résultats.}

La ponte commence assez tôt puisque vers la mi-juillet, on trouve déjà des œufs dans l'enveloppe de cire des femelles. Cependant ce n'est que vers le 4 août qu'un nombre significatif d'œufs est présent. A cette date, sur des arbres ayant une densité faible de cochenilles, on trouve 2,75 œufs pour une femelle, alors qu'on a 7,35 œufs par femelle sur des arbres portant beaucoup de cochenilles.

A la date du 22 août, cette différence existe toujours, chaque femelle a pondu un nombre d'œufs plus grand : 22,35 œufs par femelle dans le cas d'une forte population et 16,9 dans le cas d'arbres à faible population d'insectes.

L'éclosion des larves chevauche la ponte : les premiers œufs pondus donnent naissance déjà à des larves, alors que la femelle pond encore. On trouve les premières dès le 4 août, mais ce n'est que le 12 septembre que l'on dispose de nombres permettant une analyse.

Si on comptabilise ensemble le nombre des œufs et le nombre des larves présents dans l'amas cireux de la femelle, on constate que l'on obtient au 12 septembre des valeurs voisines de celles du 22 août. On peut donc penser qu'au début du mois de septembre, la ponte était terminée : le nombre des descendants (larves + œufs) dans l'amas cireux reste constant et on ne retrouve pas de larves en dehors de cet amas cireux.

Les larves commencent à quitter les enveloppes de fils cireux vers la fin septembre et ce mouvement se poursuit jusqu'au 12 octobre, date à laquelle il ne reste que très peu d'œufs dans les enveloppes.

On peut résumer ces résultats sous la forme du tableau suivant : 20 juillet 4 août 22 août 12 sept. 28 sept. 12 oct.

$\begin{array}{lllllll}\text { Faible population } \ldots \ldots & \overline{0} & \overline{2,75} & \overline{16,90} & \overline{15,65} & \overline{8,54} & \overline{4,20} \\ \text { Forte population } \ldots \ldots & 0 & 7,35 & 22,35 & 20,30 & 12,71 & 7,19\end{array}$

jusqu'au 21 août, il s'agit du nombre moyen d'œufs par femelle et après du nombre moyen toujours par femelle, des œufs et des larves.

\subsection{Discussion.}

Ces premiers résultats montrent que cette méthode de comptage livre des résultats utilisables.

On peut constater la date du début et de la fin de la ponte, la date de l'éclosion des larves. De plus, la fécondité réelle des femelles est plus importante sur les arbres porteurs d'une forte population de cochenilles. Ces arbres fournissent peut-être une meilleure nourriture aux femelles. La différence entre ces fécondités exprimées est faible (1,32 fois plus d'œufs par femelles sur les arbres à forte densité de cochenilles) mais elle s'ajoute à l'importance de la population de la génération parentale.

Par contre, la siłuation géographique ou l'âge des arbres, à la vue de ces résultats, ne semblent pas avoir d'importance.

\subsection{Méthodologie.}

\subsection{Fixation des larves}

Elle est sensiblement la même que pour l'étude de la fécondité. Sur les arbres choisis de la même façon que pour l'étude précédente, on a prélevé cette fois-ci 
dix rondelles. Ces dix rondelles étaient prélevées au hasard sur le périmètre d'un même arbre. On a étudié à chaque fois dix arbres.

Les prélèvements ont été effectués le 22 novembre, date à laquelle nous pensions que la phase ambulatoire du premier âge larvaire était terminée.

Les larves présentes sur la rondelle étaient dénombrées sous la loupe binoculaire. On a considéré que les larves dont le rostre était enfoncé dans l'écorce, étaient vivantes au moment du prélèvement. Les autres qui présentaient souvent des signes de dessiccation étaient considérées comme mortes.

\subsection{Résuliais.}

Il apparaît d'abord que sur des parcelles à âge égal et à densité de cochenilles égale, les valeurs obtenues par comptage, ne sont pas homogènes. Certaines valeurs s'écartent statistiquement des autres. Après élimination des valeurs trop excentrées, on peut comparer les résultats selon l'âge.

\section{Série II}

Série VIII

$\begin{array}{cccc}90-120 \text { ans } & 60-90 \text { ans } & 90-120 \text { ans } & 60-90 \text { ans } \\ \frac{-}{15} & -17 & - & - \\ 7 & 8 & 15 & 25 \\ & 11 & 11 & 5\end{array}$

Total des larves ..........

Larves installées ..........

A la date du 22 septembre, de nombreuses larves étaient mortes. Il faut remarquer aussi que les échantillons sont très hétérogènes entre eux sur le plan statistique. Il est donc difficile de donner un taux de réussite d'installation pour les jeunes larves ; cependant, cette réduction importante existe dans tous les cas.

\subsection{Conclusions}

Cette première étude avait pour but de.tester la méthodologie. Les comptages sous la loupe sont suffisamment précis pour fournir des données exploitables.

II apparaît qu'en 1978, les femelles faisant partie d'une population importante ont une descendance un peu plus abondante. Après la période où les larves se déplacent, le nombre des larves qui ont réussi à s'installer est bien plus faible que le nombre des larves qui sont nées. C'est une période cruciale pour les populations.

Par contre les arbres sont très hétérogènes entre eux, en ce qui concerne les populations de larves fixées. Sur un lot d'arbres où la densité des populations parentales était la même, on trouve des densités très différentes en ce qui concerne la population fille. Il faut donc reconsidérer le mode de prise d'échantillons pour mesurer la fixation des larves.

\section{II. - Evolution des populations de Cryptococcus fagi en Haute-Normandie}

En vue de suivre l'évolution des attaques de la cochenille du hêtre et de la maladie de l'écorce due à Nectria coccinea, on a implanté en 1978 un dispositif d'observations dans quatre forêts domaniales de Haute-Normandie : Brotonne, Lyons, Eawy, Eu. 
Les observations sont effectuées sur des transects linéaires permanents que vous pourrez voir lors de notre visite en forêt de Lyons. Chaque transect contient cent arbres numérotés et qui sont suivis individuellement. Dans chacun des massifs forestiers, on a installé neuf transects, répartis selon les types de sol, et pour chacun de ces types de sol, selon trois classes d'âge.

L'estimation du degré d'attaque par la cochenille et par Nectria est effectuée au mois d'octobre par les agents de l'O.N.F., selon une échelle de notation simple. L'échelle pour la cochenille est celle que nous avons déjà utilisée (Malphettes, 1977. Ann. Sci. For., 34 (2), 159-173) et l'échelle pour le Nectria a été proposée par R. Perrin.

Les emplacements de ces dispositifs n'ont pas été choisis au hasard : on a tenu compte en particulier, de l'existence de populations de cochenilles. Ils donnent donc des résultats qui sont plus élevés que la moyenne pour un massif forestier. La comparaison entre classes d'âge ef types de sol reste possible, mais il convient d'être prudent pour comparer à l'aide de ces résultats un massif à un autre. Les premières notations ont eu lieu à l'automne 1978.

\subsection{Comparaison selon les massifs forestiers}

Comparons d'abord la situation dans les trois massifs de Lyons, Eawy ef Eu pour lesquels il a été possible de retenir trois classes d'âge (perchis, futaie jeune ef vieille futaie) et trois types de sol (sol sur limon, sur limon à silex et sur craie) absolument comparables.

Sur les neuf cents arbres par massif, on a les effectifs suivants :

$\begin{array}{cc}\text { Très peu ou } & \text { Faiblement } \\ \text { pas attaqués } & \text { attaqués } \\ \text { Note } 0 & \text { Notes } 1-2\end{array}$

Lyons ...... $\quad 617(70$ p. 100)

Eu ......... $\quad 542(60$ p. 100)

Eawy ....... $\quad 458(50$ p. 100$)$
$229(25$ p. 100)

306 (34 p. 100)

378 (42 p. 100)
Fortement
attaqués
Notes 2-4-5

54 (6 p. 100)

42 (5 p. 100)

65 (7 p. 100)

La situation est donc comparable d'un massif à l'autre surtout pour les attaques fortes. On a partout le même nombre d'arbres très attaqués.

En forêt de Brotonne, les arbres faiblement attaqués sont encore plus nombreux (quatre cent soixante arbres soit 51 p. 100 avec la note 1-2 et trois cent quatre-vingtdix-sept arbres avec la note 0 , soit 44 p. 100) ; les arbres fortement attaqués ne sont par contre, pas plus abondants à Brotonne qu'ailleurs. La forêt de Brotonne n'étant pas sur les mêmes sols que les trois autres massifs, les comparaisons doivent être plus prudentes.

\subsection{Comparaison selon les types de sol}

Les attaques sur sol à base de craie sont netfement moins nombreuses ef moins fortes que sur les autres sols. Pour la note 0 , on a les répartitions suivantes : 
Craie

Limon

Limon + Silex

Brotonne ....

170 (60 p. 100)

Lyons .......

244 (81 p. 100)

Eawy ........

245 (81 p. 100)

Eu ........ 254 (84 p. 100)

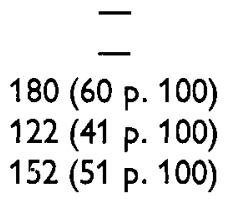

$193(64$ p. 100)

$90(30$ p. 100)

136 (45 p. 100)

Il n'y a pas de différences entre les sols sur limon ef les sols sur limon à silex. A Brotonne, les sols sont, soit sur alluvions anciennes, soit sur des argiles à silex. Les comparaisons sont donc difficiles à faire avec les autres forêts.

\subsection{Comparaison selon l'âge}

Pour les massifs de Lyons, Eu et Eawy où les arbres se répartissent entre les différentes notes et les différents âges $(P=$ Perchis, $F=$ Futaie jeune, $V F=$ Vieille futaie) selon les proportions suivantes :

\begin{tabular}{l|rrr|rrr|rrr|rrr}
\multicolumn{1}{c}{} & \multicolumn{3}{c}{ Lyons } & \multicolumn{3}{c}{ Eu } & \multicolumn{4}{c}{ Eawy } & \multicolumn{3}{c}{ Ensemble } \\
notes & P & F & VF & P & F & VF & P & F & VF & P & F & VF \\
$0 \ldots .$. & 77 & 66 & 62 & 57 & 66 & 57 & 55 & 46 & 52 & 189 & 178 & 171 \\
$1-2 \ldots$ & 18 & 32 & 27 & 40 & 29 & 37 & 31 & 53 & 42 & 89 & 114 & 106 \\
$3-4-5 \ldots$ & 5 & 2 & 11 & 3 & 5 & 6 & 14 & 1 & 6 & 22 & 8 & 23
\end{tabular}

Il n'apparaît pas d'influence de l'âge des peuplements sur l'infestation.

\section{III. - Attaques par Nectria}

- Sur les trois mille six cents arbres que comporte le dispositif dans son ensemble, cent quinze arbres portaient à l'automne 1978, des traces d'attaques par Nectria. La faiblesse de cet effectif interdit toute comparaison.

Il apparaît cependant, que l'on retrouve le plus grand nombre d'arbres porteurs de traces d'attaques de Nectria (cinquante-et-un arbres) en forêt d'Eu. La forêt d'Eawy esł beaucoup moins atteinte, alors qu'il n'y a pratiquement pas de Nectria en forêt de Brotonne. La comparaison de l'évolution des attaques de Nectria par rapport à l'évolution des colonies de Cryplococcus sera au fil des prochaines années, sans doute intéressante.

\section{IV. - Discussion}

Les dispositifs dont il est question en paragraphe 2 et 3 doivant subsister au moins une dizaine d'années.

Ces premiers relevés donnent une image de l'état de populations de Cryptococcus fagi et des attaques de Nectria coccinea au début de l'expérience, à l'automne 1978. Actuellement, il semble exister une différence d'infestation sur les sols à base de craie 
où les attaques sont bien plus faibles. L'image relevée en 1978 est le résultat d'une évolution antérieure que nous ne connaissons pas.

Par ailleurs, comme de nombreux forestiers l'ont constaté, il existe une très grande hétérogénéité dans les populations de cochenilles d'un arbre à l'autre. Cette hétérogénéité est due sans doute en partie, à la liaison qui existe entre l'insecte et son hôte. Nous entreprenons donc l'étude de l'écorce du hêtre qui est l'interface entre la cochenille et l'arbre. Ces travaux qui en sont à une phase préliminaire devraient montrer les facteurs qui peuvent conduire à une non-colonisation par la cochenille. Si ce facteur n'explique pas suffisamment la relation entre l'hôte et l'insecte il conviendra de s'intéresser à la qualité de la nourriłure, c'est-à-dire aux cellules explorées réellement par la cochenille et à leur contenu.

Reçu pour publication en décembre 1979.

\section{Summary}

\section{Undertaking in vestigations on Cryptococcus fagi Baer in Haute-Normandie}

The author discusses the first results of studies on the dynamics of the Beech scale.

Fertility fluctuates with population density of the scale on the stem : fertility is high with high level density.

The number of settled crawlers is much smaller than that of the crawlers present on the same area of bark.

One experiment was set in 1978 to observe population evolution of this scale in Haute-Normandie during the following years. It appears that the population density is the smallest when the soil is calcareous.

\section{Zuzammenfassung}

Der Verfasser legł für Diskussion seine erste Ergebnisse der Studien hinsichtlich der Dynamik der Buchenwollschildlaus vor.

Je mehr wichtig ist die Bevölkerungsdichte auf dem Stamm, desto mehr gross ist die Fruchtbarkeit der Schildiaus ist.

Die Anzahl der an der Rinde jungen angehefteten Schildlaüse isł deutlich niedriger, als die Anzahl der auf der selbe Rindenfläche anwesenden Larven.

In 1978 wird eine Erfahrungsanlage eingeführt, um in der Haute-Normandie im Laufe der nächsten Jahren auf die Entwicklung der Populationen dieser Laus zu folgen. Zur Zeit scheint es, dass die Bevölkerungsdichte, in dem selben Buchenwald, sehr geringer ist, wenn der Boden kalkhaltig ist. 